\title{
Howdy, partner!
}

DOI:

10.1038/nri2146
A recent study published in Immunity has shown that the T-cell co-stimulatory molecules B7.1 (also known as CD80) and programmed cell death 1 ligand 1 (PDL1) are binding partners, thereby revealing a new means of regulating $\mathrm{T}$-cell responses.

The interaction of co-stimulatory molecules B7.1 and B7.2 (also known as CD86) on the surface of antigenpresenting cells with receptors CD28 and cytotoxic T-lymphocyte antigen 4 (CTLA4) on the surface of T cells is essential for the generation and regulation of robust adaptive immune responses. This pathway of the B7-CD28 family of co-stimulatory molecules is well characterized but complex, as B7.1 and B7.2 have dual specificity for the stimulatory CD28 receptor and the inhibitory CTLA4 receptor. One of the newer identified pathways of this family comprises the PD1 receptor and its two ligands PDL1 and PDL2. The roles of PDL1 and PDL2 in T-cell activation have not yet been fully elucidated.

Previous studies have shown evidence for B7-dependent responses in T cells that lack both CD28 and CTLA4, and these findings, together with conflicting reports of both stimulatory and inhibitory functions for PDL1 and PDL2, prompted the authors to consider that there may be an additional functional receptor for B7.1 or B7.2 on T cells. Using a cDNA library made from Cd $28^{-1-} \mathrm{Ctla}^{-/-} \mathrm{CD} 4^{+} \mathrm{T}$ cells, the authors identified PDL1 as a ligand for B7.1. Surface plasmon resonance characterized the affinity of the B7.1-PDL1 interaction and indicated that B7.1 interacts more strongly with PDL1 than with CD28 but less strongly than with CTLA4, and that PDL1 interacts less strongly with B7.1 than with PD1. To identify the B7.1-PDL1 interaction site, Butte et al. used protein crosslinking and mass spectrometry and found that there was partial overlap of the B7.1-PDL1 interface with those of B7.1-CTLA4 and PD1-PDL1.

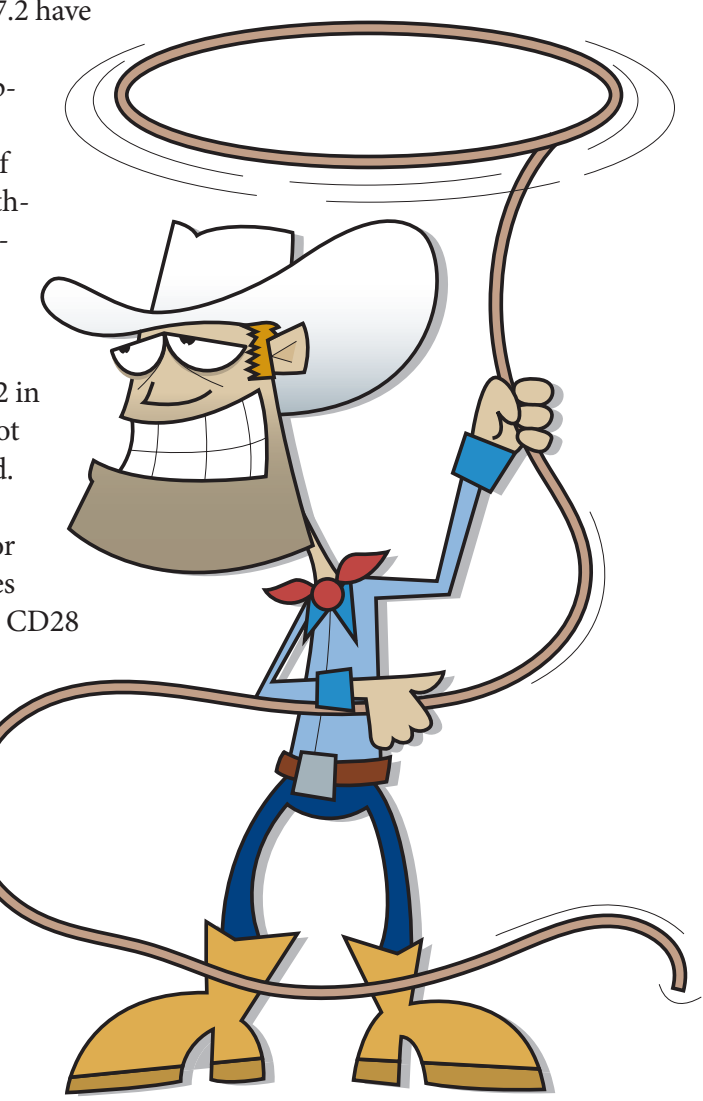

This suggests that CTLA4, CD28 and PDL1 could compete for binding to B7.1, and that B7.1 and PD1 could compete for binding to PDL1.

Next, the authors investigated the functional significance of the interaction between B7.1 and PDL1 in vitro. B7.1-coated beads delivered an inhibitory signal to Cd28 $8^{-/-}$tla $^{-/-} \mathrm{T}$ cells, resulting in a decrease in proliferation, cytokine production and activation-marker expression. In vitro proliferation of PD1-deficient T cells was inhibited by PDL1-coated beads. Finally, to confirm the specificity of the B7.1-PDL1 interaction, responses of $P d 1^{-/-} \mathrm{T}$ cells versus $P d 1^{--} C d 80^{-1-} \mathrm{T}$ cells to PDL1, and of $\mathrm{Cd} 28^{-/-} \mathrm{Ctla4}^{-/-} \mathrm{T}$ cells versus Cd28 ${ }^{-1-}$ Ctla4 $^{-1-}$ Pdl1 ${ }^{-1-}$ T cells to B7.1 were measured. The results showed that B7.1 acts specifically through PDL1 to inhibit T-cell proliferation in the absence of CD28 and CTLA4, and that PDL1 can exert an inhibitory effect on T cells either through B7.1 or PD1. Therefore, there is a specific and significant bidirectional interaction between B7.1 and PDL1 that inhibits T-cell responses.

This previously unsuspected interaction indicates increased functional significance of B7.1 and PDL1 on $\mathrm{T}$ cells, and means that the roles of B7.1 and PDL1 in regulating the activation and inhibition of immune responses need to be reassessed. These findings also have implications for the use of these molecules as therapeutic agents.

Sharon Ahmad 